\title{
NITRIC OXIDE FORMATION DURING FLAME/VORTEX INTERACTION
}

\author{
VITO S. SANTORO, DIMITRIOS C. KYRITSIS, MITCHELL D. SMOOKE AND ALESSANDRO GOMEZ \\ Yale Center for Combustion Studies \\ Department of Mechanical Engineering \\ Yale University \\ New Haven, CT 06520-8286, USA
}

\begin{abstract}
The formation of nitric oxide was investigated in methane diffusion flames under the perturbation of laminar vortices. Laser-induced fluorescence and Raman spectroscopy were used to measure nitric oxide, major species, and temperature, and to obtain the time evolution of the scalar dissipation rate at the stoichiometric surface during the flame/vortex interaction. Vortices with two characteristic times were thrust into the flame and their effects were compared with steady flames at the same scalar dissipation rate. The experimental measurements showed that the vortex did not affect significantly the peak temperature, $\mathrm{CO}$, and $\mathrm{CO}_{2}$ mass fraction during the interaction. $\mathrm{NO}$, instead, was strongly affected. The peak mass fraction of $\mathrm{NO}$ in flames under vortex perturbation differed by almost a factor of 2 from steady flames with similar scalar dissipation rates. One-dimensional numerical simulations, used under the well-justified assumption that the vortices induced negligible curvature, yielded results in good agreement with the experimental measurements. The numerical computations were also used to investigate the effect of the characteristic time of the vortex on the different NO formation paths, that is, thermal, and prompt. The peak mass fraction of thermal NO was shown to be strongly dependent on the timescale of the unsteady perturbation, while prompt NO was almost independent. The emission index, that is, the production of NO normalized by the consumption of the fuel, behaved quasi-steadily for the two formation paths. The results were rationalized by considering the flame structure and the activation energy of the different formation paths.
\end{abstract}

\section{Introduction}

Pollutant formation in combustion processes is a topic of growing public concern and subject to increasingly stringent regulations. As a result, a significant experimental and numerical effort has been devoted to the subject in recent years. To circumvent the intrinsic complexity of turbulent flames and consequent limitations in experimental and computational capabilities to treat these types of flows, laboratory research has often focused on steady laminar flames. Here, a situation of intermediate complexity is investigated, namely, the interaction of conterflow flames with laminar vortices. A review of the work done on this subject is presented in Ref. [1]. Specifically, this approach proved to be useful for the quantitative study of extinction phenomena in diffusion flames [2-4]. This work showed that extinction is not dependent on the timescale of the perturbation when described in terms of the scalar dissipation rate at the stoichiometric surface, because of the fast chemistry associated with the heat release. By the same assumption, flamelet models have been used to predict the structure of turbulent diffusion flames. Attempts to predict pollutant formation, on the other hand, have been more problematic, especially because the timescales involved in the chemistry responsible for the formation of major pollutants are slow relative to the relevant fluid dynamic timescales. For example, Pitsch et al. [5] had to resort to an unsteady flamelet model to compute nitric oxide in turbulent flames, to reach agreement with the experimental measurements within $25 \%-50 \%$.

Nitric oxide has been measured by different experimental techniques and has been computed in steady one-dimensional and two-dimensional diffusion flames. For instance, Smooke et al. [6] measured nitric oxide in jet diffusion flames via laserinduced fluorescence (LIF) and compared the experimental results with numerical computation, showing good agreement between the two. Li and Williams [7] measured $\mathrm{NO}_{x}$ in counterflow diffusion flames using gas chromatography and compared the experimental results with numerical simulations. Counterflow diffusion flames with time-dependent boundary conditions offer yet another controlled system in which numerical simulations have been performed [8]. This work showed the effect of the oscillating frequency on the production of $\mathrm{NO}, \mathrm{NO}_{2}$, $\mathrm{CO}$, and the time delay generated by the unsteady boundary conditions. On the other hand, pollutant formation under unsteady perturbations has received little attention from an experimental standpoint. An interesting experimental work was carried 
out in Ref. [9], in which soot formation in counterflow diffusion flames was investigated under oscillating velocities at the boundary.

In the present work, the production of nitric oxide under a vortex-induced perturbation is studied. In particular, the influence of the characteristic timescale of the vortex on the formation of NO is investigated in detail and the result compared with steady flames with the same scalar dissipation rate. Onedimensional time-dependent computations are compared with the experimental measurements to shed light on the different NO formation paths and their sensitivity to unsteady perturbations.

\section{Experimental Apparatus}

Details of the burner apparatus were reported in Ref. [2]. In brief, an axisymmetric counterflow diffusion flame was established in a vertical configuration, with the oxidizer being fed from the top, $\mathrm{CH}_{4}$ and $\mathrm{N}_{2}$ from the bottom. Both sides of the burner terminated in a contraction with an exit diameter of $12.5 \mathrm{~mm}$ and a separation distance of $13 \mathrm{~mm}$. A steady flame, with mass fraction of the fuel, $Y_{\mathrm{f}}=$ 0.12 , on the fuel side and mass fraction of $\mathrm{O}_{2}$, $\mathrm{Y}_{\mathrm{Ox}}=1.0$, on the oxidizer side, was perturbed by periodically generated laminar toroidal vortices from the oxidizer side. The boundary conditions were chosen to have a relatively high flame temperature, and consequently, produce a significant amount of NO. At the same time, soot production, which would have caused significant complications with the experimental measurements, was avoided. Vortices of different characteristic timescales, with a characteristic dimension much larger than the thickness of the mixing layer, were generated to mimic unsteady perturbations characteristic of turbulent environment.

Nitric oxide was measured using LIF. The third harmonic of a Nd:YAG laser pumped a dye laser using Coumarin 450. The laser was tuned to $452 \mathrm{~nm}$ and its output was doubled to produce the 225.8-nm excitation (4 mJ/pulse at $10 \mathrm{~Hz}$ repetition rate). The UV beam was focused by a 20-cm-focal-length quartz lens on the burner centerline. Spectral features over a wavelength range from 225.4 to $226.9 \mathrm{~nm}$ were matched with the data of Engleman et al. [10]. The $Q_{1}(18)$ transition of the $A^{2} \Sigma^{+}-$ $X^{2} \Pi(0,0)$ band $\left(44,275.684 \mathrm{~cm}^{-1}\right)$ was selected because it is well separated from neighboring transitions and it has a significant population from room temperature to flame temperature. No significant spectral interferences were encountered in the flames investigated [11]. The fluorescence signal was collected using a pair of quartz lenses (8-cm focal length) and it was imaged onto the $300-\mu \mathrm{m}$ entrance slit of a spectrometer. The fluorescence was dispersed into a 300-groove/mm grating and imaged with an intensified CCD camera (Apogee AP7).
Spectrally, the region from 230 to $270 \mathrm{~nm}$ was recorded, including the $(0,1),(0,2)$, and $(0,3)$ vibrational fluorescence bands. The fluorescence signal was integrated on the camera chip for $2 \mathrm{~s}$, equivalent to 20 laser shots, and only the $(0,2)$ fluorescence band was used for the measurements. The fluorescence experiment was operated in the linear regime. The concentrations of the quenching partners and temperature were measured using Raman scattering, as described below, and the appropriate corrections were applied following Refs. [12,13].

The LIF measurements were complemented with Raman scattering measurements. A detailed description of this technique in this experimental configuration was presented in Ref. [3]. A Nd:YAG laser delivering $100 \mathrm{~mJ} /$ pulse at $532 \mathrm{~nm}$ was used as the excitation source. The laser beam was focused to an approximately $200 \mu \mathrm{m}$ thick and $30 \mathrm{~mm}$ long horizontal line by a $750 \mathrm{~mm}$ plano-convex lens. The horizontal line was imaged vertically on the $400-\mu \mathrm{m}$ entrance slit of an Acton Research $300 \mathrm{~mm}$ imaging spectrometer. A 50 mm, f 1.8 AF Nikkor lens was used for signal collection. To reject stray light from the burner hardware, an OG 550, $3 \mathrm{~mm}$ thick glass filter was mounted in front of the collecting lens. Dispersion was achieved with a $68 \mathrm{~mm} \times 68 \mathrm{~mm}$ ruled grating with 1200 grooves/mm and a $500 \mathrm{~nm}$ blaze wavelength. The spectrograph output was imaged on the photocathode of a DEP XX1450DH image intensifier coupled to an Apogee AP7 camera by two $50 \mathrm{~mm}$, f $1.8 \mathrm{AF}$ Nikkor lenses.

Raman measurements were used to obtain the number density of the major species $\left(\mathrm{CH}_{4}, \mathrm{O}_{2}, \mathrm{~N}_{2}\right.$, $\mathrm{H}_{2} \mathrm{O}, \mathrm{CO}, \mathrm{CO}_{2}$ ) along the burner centerline. The temperature profile and the scalar dissipation rate profiles could be obtained from the measurement of the major species. The vortex-actuating signal was used as a master pulse for the proper synchronization of both experimental systems.

\section{Numerical Approach}

Curvature effects are generally modest in hydrocarbon diffusion flames. Such effects were deliberately minimized in the present experiments using vortices much larger than the characteristic thickness of the mixing layer. The curvature was measured using vortex visualizations, similarly to Ref. [2]. The thickness of the mixing layer, $\delta_{\mathrm{m}}=\sqrt{D / A}$, was estimated based on the thermal diffusivity $D$ and the strain rate $A$. Throughout the flame/vortex interaction, the ratio of the radius of curvature to the thickness of the mixing layer was larger than 10, enabling us to neglect two-dimensional effects. Based on this simplification, the flame behavior along the stagnation streamline could be modeled as a onedimensional, time-dependent counterflow diffusion flame. The numerical simulation of the counterflow 

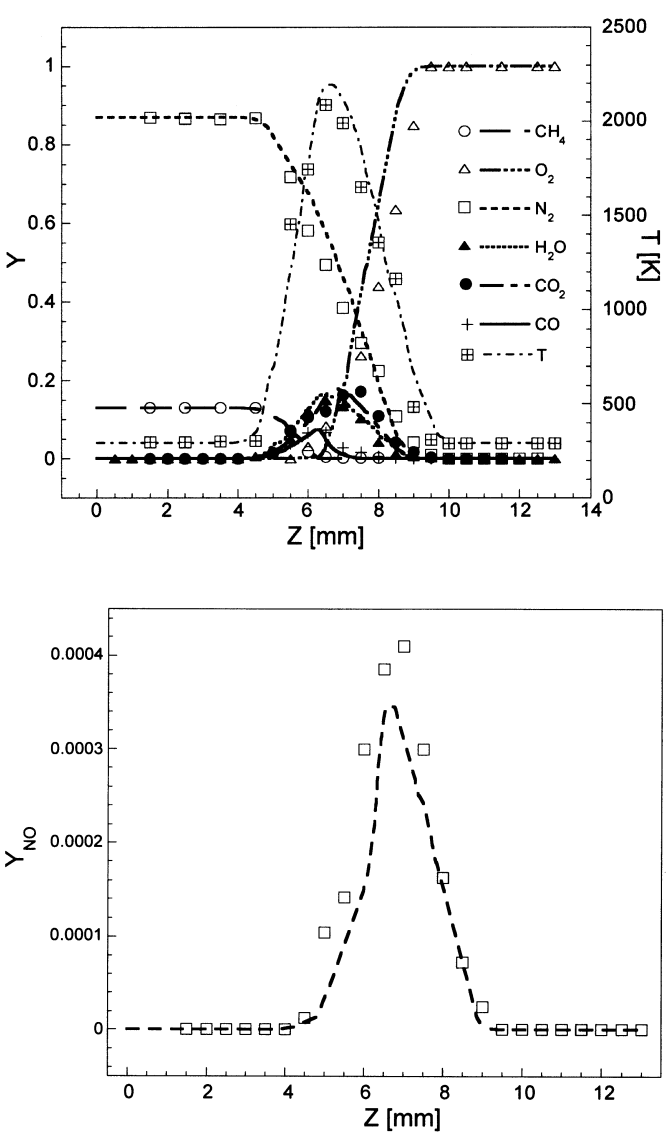

Fig. 1. (a) Experimental (symbols) and computed (line) major species mass fractions (left ordinate), and temperature (right ordinate) along the centerline for a steady flame. (b) Nitric oxide mass fraction as function of the axial coordinate from experimental measurements (symbols) and computation (line).

diffusion flame was obtained solving the mass, momentum, energy, and species conservation equations along the stagnation streamline using a model described in Ref. [14], where the pertinent conservation equations and the employed boundary and initial conditions are described in detail. An existing numerical code [14], developed to simulate steady counterflow diffusion flames, was modified by introducing a backward Euler approximation for the evaluation of the time derivatives in the conservation equations. The numerical model used detailed chemical kinetics (GRI version 2.11 [15], 48 species and 275 reactions), and transport properties. GRI 2.11 was chosen as the mechanism yielding the best agreement with the experimental data. The initial solution for the time-dependent computations was obtained using the steady-state model, with the experimentally measured boundary conditions. The

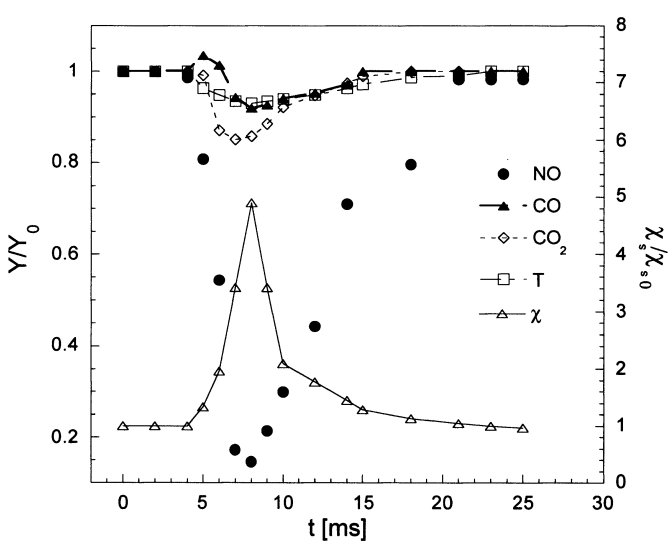

FIG. 2. Time evolution of experimentally measured peak mass fraction of $\mathrm{NO}, \mathrm{CO}, \mathrm{CO}_{2}$, peak temperature (left ordinate), and scalar dissipation rate at the stoichiometric surface (right ordinate), normalized by the initial value.

time-dependent calculations were then performed imposing a velocity perturbation at the oxidizer side boundary, which was obtained from experimental LDV measurements, as in Ref. [2].

\section{Results and Discussion}

The spatial profiles of the major species $\left(\mathrm{CH}_{4}, \mathrm{O}_{2}\right.$, $\mathrm{N}_{2}, \mathrm{H}_{2} \mathrm{O}, \mathrm{CO}_{2}, \mathrm{CO}$ ), measured with Raman scattering along the centerline of the burner, are presented in Fig. la (symbols) for a vortex-perturbed flame at the beginning of the interaction (left ordinate). The experimental measurements are compared to numerical simulations (lines), showing good agreement. In the same figure, the temperature profile (right ordinate), obtained from the total number density of the measured species, is also reported. Fig. 1b shows the experimental measurements of NO (symbols) and the results of the numerical simulations (lines). The two profiles compare well with a difference between the peak NO mass fractions of less than $30 \%$. The species profiles were used to estimate the quenching corrections for NO, similarly to Ref. [6], and to obtain the scalar dissipation rate at the stoichiometric surface, as in Ref. [3]. The latter was defined as $\chi_{\mathrm{s}}=2 D|\nabla Z|_{s}^{2}$ where $D$ is the thermal diffusivity of the mixture, and $Z=(\beta-$ $\left.\beta_{\mathrm{ox}}\right) /\left(\beta_{\mathrm{f}}-\beta_{\mathrm{ox}}\right)$ the mixture fraction (ox and $\mathrm{f}$ indicate the conditions on the oxidizer side and the fuel side, respectively). The conserved scalar $\beta$ was defined as $\beta=Y_{N_{2}}$, for reasons explained in Ref. [3]. Evidently, this definition is based on the presumed inertness of $\mathrm{N}_{2}$ and does not take into account the $\mathrm{NO}_{x}$ chemistry. Numerical simulations showed this approximation to be very accurate.

Figure 2 shows experimental measurements of the time evolution of peak $\mathrm{NO}, \mathrm{CO}, \mathrm{CO}_{2}$ mass fraction 


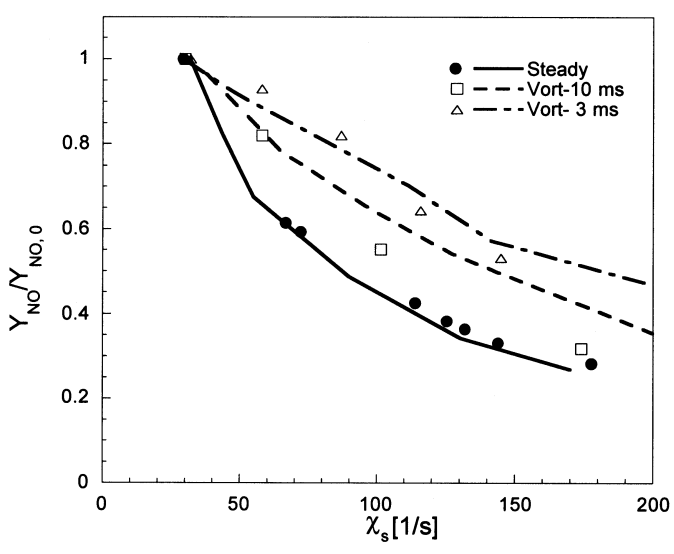

FIG. 3. Peak mass fraction of NO for steady and vortexperturbed flames normalized by their initial value. Experimental measurements (symbols) and numerical simulations (lines) are reported as a function of the scalar dissipation rate at the stoichiometric surface.

and temperature (left axis) during the flame/vortex interaction. Also shown in the same figure is the time evolution of the scalar dissipation rate at the stoichiometric surface (right axis). All quantities are normalized by their initial values. The vortex, generated every $100 \mathrm{~ms}$, started its interaction with the flame $4 \mathrm{~ms}$ after its actuation. The interaction lasted almost $15 \mathrm{~ms}$, during which the peak mass fraction of nitric oxide decreased by more than $90 \%$ with respect to its initial value, while the scalar dissipation rate increased five times, before recovering its original value $\left(\chi_{0}=301 / \mathrm{s}\right)$. The peak temperature was mildly affected by the change in scalar dissipation rate. However, even mild change in peak temperature can result in significant changes in the contribution from thermal NO. The peak mass fraction of carbon dioxide varied less than $15 \%$ during the interaction and the peak mass fraction of carbon monoxide first increased above the initial value, then decreased by an amount smaller than $10 \%$ before recovering the initial conditions. A similar trend in CO was observed in Ref. [8] and in our own numerical simulations.

The effect of the characteristic vortex turnover time on the formation of $\mathrm{NO}$ is presented in Fig. 3. Such a time was estimated as $t_{\mathrm{vor}}=\pi(d / 2)^{2} / \Gamma$, where $d$ is the size of the vortex core, and $\Gamma$ the circulation. This timescale was varied changing the circulation of the vortex, and more specifically, changing the rising time and the amplitude of the voltage function used to generate the vortex. The circulation of the vortex together with the main parameters of the vortex, core size, vortex size, translational velocity, were measured experimentally using laser Doppler velocimetry (LDV), similarly to
Ref. [4]. The timescale of the vortex for these experiments was chosen so as to induce time-dependent effects on the flame without causing extinction. As a result, the characteristic timescale of the vortex had to be smaller than the diffusion time of the flame in steady conditions, $\tau_{\text {diff }}=1 / A_{\mathrm{ss}}$, where $A_{\mathrm{ss}}$ is the steady-state strain rate, and larger than the characteristic timescale of extinction, $\tau_{\text {ext }}=1 / A_{\text {ext. }}$. As a result, for the present experiment, the vortex time had to satisfy the following inequality, $1 \mathrm{~ms}<\tau_{\text {vor }}<$ $25 \mathrm{~ms}$. Two different vortices with time scales of $\tau_{\text {vor }}=3 \mathrm{~ms}$ and $\tau_{\text {vor }}=10 \mathrm{~ms}$ were generated. Fig. 3 shows the variation of the peak NO introduced by the two vortices as a function of the scalar dissipation rate at the stoichiometric surface for the first part of the interaction, in which the scalar dissipation rate increases. Measurements of the second part of the interaction are omitted simply for clarity of presentation. In the same figure, the peak of NO for steady flames is reported in the same range of scalar dissipation rates. The latter was increased by increasing the exit velocity on both sides of the flame. These experimental measurements (symbols) are compared to the numerical simulation (lines). In view of the discrepancy in peak mass fraction observed in Fig. 1b, all quantities are normalized with the values at a reference scalar dissipation rate to compare relative trends. Two main features can be observed from both experimental results and numerical computations. First, the peak nitric oxide decreases for both steady and unsteady flames as the scalar dissipation increases.

Second, the peak mass fraction of nitric oxide depends on the characteristic timescale of the vortex for a given scalar dissipation rate. The peak mass fraction of $\mathrm{CO}, \mathrm{CO}_{2}$, and peak temperature, instead were mildly affected by the variations in scalar dissipation rate, as shown in Fig. 2, and they were almost unaffected by the characteristic timescale of the vortex.

To determine the relative contribution of the main paths to NO formation, namely, thermal, prompt, $\mathrm{N}_{2} \mathrm{O}$ route, and through the reaction $\mathrm{O}+\mathrm{NNH}=$ $\mathrm{NH}+\mathrm{NO}$ [16], additional numerical simulations were performed. The last two formation paths provide a negligible contribution to the amount of total NO under the present conditions and are omitted from the discussion that follows. Thermal NO was calculated using the hydrocarbon part of the mechanism [15] and appending only the thermal portion of the $\mathrm{NO}_{x}$, similarly to Ref. [6]. The contribution of "prompt" NO was evaluated as a difference of the amount calculated with the full chemistry and the one with thermal NO.

In Fig. 4, the results of thermal NO and prompt $\mathrm{NO}$ are reported as a function of the scalar dissipation rate for both steady and vortex-perturbed flames. We observe that thermal NO decreases abruptly for larger values of the scalar dissipation 


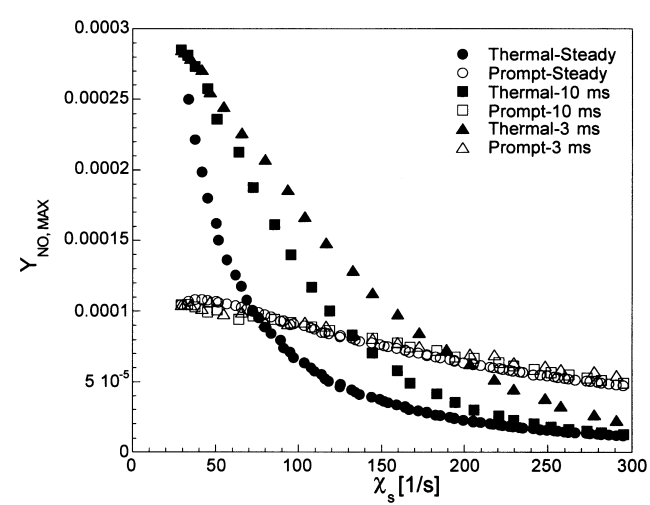

FIG. 4. Experimental (symbols) and computed (line) peak mass fraction of thermal and prompt NO reported as a function of the scalar dissipation rate at the stoichiometric surface for flames under unsteady perturbations with characteristic timescale of 3 and $10 \mathrm{~ms}$ and under steady conditions.

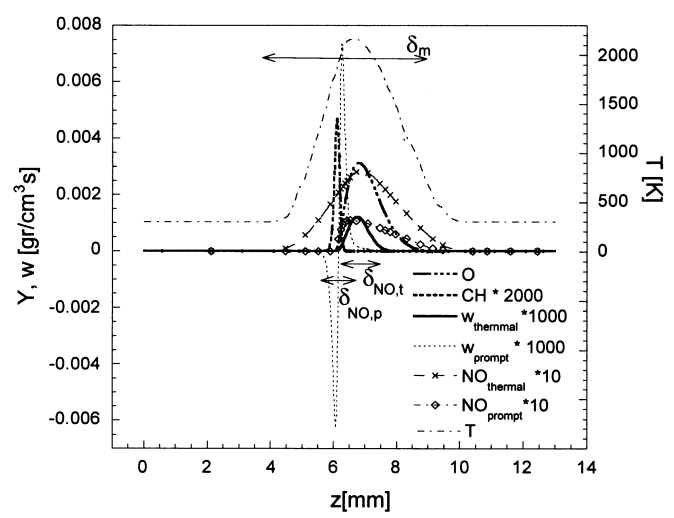

Fig. 5. (Left ordinate) Computed species mass fraction of $\mathrm{O}, \mathrm{CH}$, thermal $\mathrm{NO}$, and prompt $\mathrm{NO}$, and chemical source terms for thermal and prompt NO, along the centerline of a steady flame with scalar dissipation rate. (Right ordinate) Temperature versus axial coordinate.

rate. This can be explained in terms of the residence time. For small values of the scalar dissipation rate, the residence time is large and the formation of $\mathrm{NO}$ through the thermal path is favored due to higher temperatures. The characteristic timescale of the vortex affects the peak thermal NO significantly. The maximum variation between the steady and the vortex-perturbed flames reaches almost 200\%. The prompt mechanism is mildly affected by the change in scalar dissipation rate and the characteristic timescale of the vortex, at a level of less than $10 \%$. The results for prompt $\mathrm{NO}$ are consistent with the numerical simulation of Im et al. [8], in which the NO present in the system was mainly "prompt".
The behavior of the peak mass fraction with the timescale of the perturbation can be interpreted on the basis of the flame structure and characteristic timescales involved in the process. The structure of an unsteady diffusion flame has been described as consisting of an outer zone in which a balance of convection, diffusion, and accumulation takes place and a quasi-steady diffusive-reactive inner layer that has been described by rate-ratio asymptotics [17]. The latter, in turn, consists of a fuel consumption zone, a radical equilibration zone, and an oxygen consumption zone. Fig. 5 shows computed profiles along the flame axis of the mass fractions of $\mathrm{O}, \mathrm{CH}$, thermal NO, prompt $\mathrm{NO}, \mathrm{T}$, and the production rates of thermal and prompt NO for a flame with scalar dissipation rate equal to $30 \mathrm{~s}^{-1}$. Thermal NO is produced in a thin layer, the oxidation zone, where the mass fraction of $\mathrm{O}$ is significant and the temperature changes with respect to the peak temperature by an amount of order $R T_{\mathrm{f}}^{2} / E_{\mathrm{NO}}$, where $E_{\mathrm{NO}}$ is the activation energy for the initiation step $\mathrm{N}_{2}+\mathrm{O}$ $\rightarrow \mathrm{NO}+\mathrm{N}$, and $T_{\mathrm{f}}$ the flame temperature. Thermal NO diffuses outside this production layer and can be found in appreciable concentrations throughout most of the outer layer as well, which, for the present purpose, can be taken as the region in which $T$ departs from its value at the boundaries. Prompt NO, on the other hand, is formed in the even thinner fuel consumption zone, in which the concentration of $\mathrm{CH}$ is significant. Fig. 5 shows that it is consumed (reburn) on the fuel side, as the negative production rate indicates, and diffuses only on the oxidizer side, in a region relatively thinner than that in which thermal NO is present.

To explain the different behavior of various species under unsteady perturbation, consider the species mass fraction governing equation for the generic species $Y$ in the unsteady diffusive reactive inner layer of thickness $\delta_{\mathrm{RL}}$ :

$$
\frac{\partial Y}{\partial t}=\omega_{Y}-\frac{\partial}{\partial z}\left(D \frac{\partial Y}{\partial z}\right) \approx \omega_{Y}-\frac{D}{\delta_{\mathrm{RL}}^{2}} Y
$$

where $\delta_{\mathrm{RL}} \approx \varepsilon \delta_{\mathrm{m}}=\varepsilon \sqrt{D / \chi}$ with $\varepsilon$ being a small quantity, of the order of the inverse of a Zeldovich number, and $\delta_{\mathrm{m}}$ is the thickness of the mixing layer. In the limit of $(\partial Y / \partial t) \rightarrow 0$, equation 1 becomes

$$
0 \cong \omega_{Y}-\frac{D}{\delta_{\mathrm{RL}}^{2}} Y=\omega_{Y}-\frac{\chi Y}{\varepsilon^{2}}=\omega_{Y}-\frac{Y}{\tau_{\mathrm{ch}}}
$$

with $Y \rightarrow Y_{\mathrm{s}}=\tau_{\mathrm{ch}} \omega_{Y}, \tau_{\mathrm{ch}}$ being a characteristic chemical time for the generic species $Y$. Subtracting equation 2 , with $Y=Y_{\mathrm{s}}$ from equation 1 , yields a

$$
\begin{aligned}
\frac{\partial}{\partial t}\left(Y-Y_{\mathrm{s}}\right) & +\frac{1}{\tau_{\mathrm{ch}}}\left(Y-Y_{\mathrm{s}}\right)=-\frac{\partial Y_{\mathrm{s}}}{\partial t} \\
& =-\frac{\partial Y_{\mathrm{s}}}{\partial T} \frac{\partial T}{\partial t}=-Y_{\mathrm{s}} \frac{E}{R T} \frac{1}{T} \frac{\partial T}{\partial t}
\end{aligned}
$$

in which the last equality is obtained from the expression of $Y_{\mathrm{S}}$ by assuming Arrhenius kinetics for the 


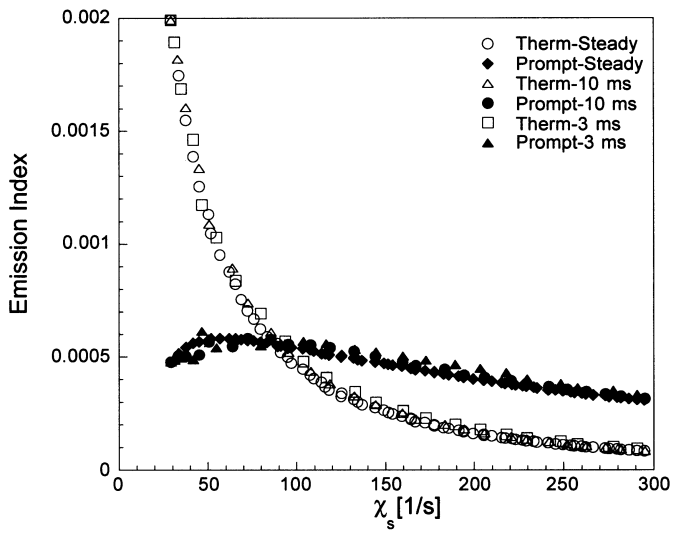

FIG. 6. Computed emission index for thermal and prompt NO for steady and vortex-perturbed flames as a function of the scalar dissipation rate at the stoichiometric surface.

reaction rate. For large activation energy processes, such as that concerning thermal NO, significant forcing may be observed on the right-hand side of equation 3. Other species with smaller activation energy, on the other hand, may approach a quasi-steady behavior since the right-hand side of equation 3 is small. For such species, $Y$ exponentially relaxes to $Y_{\mathrm{s}}$ in a short time, $\tau_{\text {ch }}$.

The relative variation between unsteady flames and steady flames can be now estimated. If the chemical time associated with the production of the generic species $Y$ is much smaller than the unsteady time, as is typically the case, the first term in equation 3 is negligible as compared with the second, and equation 3 yields

$$
\frac{Y-Y_{\mathrm{s}}}{Y_{\mathrm{s}}} \approx-\frac{\tau_{\mathrm{ch}}}{\tau_{\mathrm{uns}}} \frac{E}{R T} \frac{\Delta T}{T}
$$

with $\tau_{\text {uns }}$ as the timescale of the unsteady perturbation, equal to $\tau_{\text {vor }}$ in this specific case. Equation 4 reveals that unsteady effects on the peak mass fraction are inversely proportional to the timescale of the perturbation, and directly proportional to the activation energy $E$, the characteristic chemical time and the temperature variation induced by the vortex, which can be construed as a measure of the strength of the perturbation.

For nitric oxide, the activation energy for thermal $\mathrm{NO}$ and prompt NO are $290 \mathrm{~kJ}$ and $90 \mathrm{~kJ}$, respectively [17], whereas the characteristic chemical timescales, estimated from the computations as $\tau_{\mathrm{ch}}=$ $\left(Y / \omega_{Y}\right)$, are $160 \mu \mathrm{s}$ and $20 \mu \mathrm{s}$, respectively. If unsteady effects induced by the vortex in the two formation paths are compared, the relative variation of the peak mass fraction with respect to the steady value of thermal NO are predicted to be 25 times more pronounced as compared with the prompt
NO. Unsteady effects are important even for NO produced from $\mathrm{N}_{2} \mathrm{O}$, since its overall activation energy is $150 \mathrm{~kJ} \mathrm{[17]} \mathrm{and} \mathrm{its} \mathrm{chemical} \mathrm{timescale} \mathrm{is} 50 \mu \mathrm{s}$. Using the scaling argument presented above, unsteady effects can be predicted to be five times more pronounced than for prompt NO. These values are consistent with the results of the numerical simulations yielding ratios of 20 and 6 . Similarly, from equation 4 we expect the significance of the unsteady effects to diminish as the timescale of the perturbation increases, as observed in Figs. 3 and 4 .

Turning to $\mathrm{CO}$ and $\mathrm{CO}_{2}$, we find activation energy values of $37 \mathrm{~kJ}$ and $15 \mathrm{~kJ}$, respectively [18], whereas the chemical times are estimated at $30 \mu \mathrm{s}$ and $135 \mu \mathrm{s}$, respectively. $\mathrm{CO}$ is produced in the fuel-consumption zone; it is consumed on the oxygen side and diffuses on the fuel side. $\mathrm{CO}_{2}$ is produced in the oxygen-consumption zone and diffuses on both the fuel and oxygen side. Unsteady effects are minimal for the mass fraction of species produced and nearly consumed in the reaction layer, such as $\mathrm{CO}$ and prompt NO, while they are more pronounced for species that can diffuse in the thicker outer layer, such as thermal $\mathrm{NO}$ and $\mathrm{CO}_{2}$.

If the other limiting case of $\tau_{\text {uns }} \ll \tau_{\text {ch }}$ of equation 4 can also be examined,

$$
\frac{Y-Y_{\mathrm{s}}}{Y_{\mathrm{s}}} \rightarrow \frac{E_{Y}}{R T} \frac{\Delta T}{T}
$$

which implies that the unsteady effects will depend only on the strength of the perturbation and the activation energy. This case, however, is not particularly interesting since the flame would extinguish well before this condition is achieved.

The emission index, calculated as [19]:

$$
E I_{\mathrm{NO}}=-\frac{W_{\mathrm{NO}} \int_{-\infty}^{+\infty} \omega_{\mathrm{NO}} \mathrm{d} y}{W_{\mathrm{CH}_{4}} \int_{-\infty}^{+\infty} \omega_{\mathrm{CH}_{4}} \mathrm{~d} y}
$$

where the integration is performed along the axial coordinate and is shown in Fig. 6 for all flames, with separate contributions due to thermal NO and prompt NO. The EI is affected only by the scalar dissipation rate, similar to the results in Ref. [20], but does not depend on the temporal history of how that particular value of $\chi$ is reached. Its quasi-steady behavior is consistent with the previously described flame structure, since the integrated chemical source terms are significant only in the quasi-steady diffusive-reactive inner layer. Furthermore, we observe that EI for thermal NO decreases abruptly with the scalar dissipation rate, while the prompt NO contribution increases first and then decreases for larger values of the scalar dissipation rate. The non-monotonic behavior of the prompt NOEI was also found in Ref. [20] for methane/air flames. The present 
analysis is consistent with experimental evidence on NO emission in practical devices, such as, for example, the dependence of $\mathrm{NO}$ emission on rotational speed for an internal combustion engine [21].

\section{Conclusions}

The formation of nitric oxide was investigated both experimentally and computationally in methane diffusion flames at various scalar dissipation rates that were established either quasi-steadily or via laminar vortex-induced perturbations. Experiments and computations were found to be in good agreement. The effect of the characteristic timescale of the vortex on the different NO formation paths was also studied computationally. Prompt NO mass fraction and emission index were found to depend only on the scalar dissipation rate, regardless of whether the flame had been strained quasi-steadily or by a vortex. The peak mass fraction of thermal NO, on the other hand, was shown to be affected by both scalar dissipation rate and its time history. CO, $\mathrm{CO}_{2}$, and the peak temperature were mildly perturbed by the presence of the vortex, while the instantaneous peak of nitric oxide was strongly affected. The results were discussed on the basis of considerations of flame structure and the different activation energies of the various chemical pathways. In the context of flamelet models, these results suggest that steady-state flamelet libraries can be used to determine the NO emission index, but not the spatial distribution of its mass fraction which is strongly influenced by unsteady phenomena.

\section{Acknowledgments}

The support of NSF grant CTS-9904296 (Dr. Farley Fisher, Contract Monitor), is gratefully acknowledged. The authors wish to thank Professors Marshall Long, Amable Liñan and Forman Williams for helpful discussions and FW in particular for the derivation of equation 3.

\section{REFERENCES}

1. Renard, P. H., Thévenin, D., Rolon, J. C., and Candel, S., Prog. Energy Combust. Sci. 26:225 (2000).
2. Santoro, V. S., Kyritsis, D. C., Liñán, A., and Gomez, A., Proc. Combust. Inst. 28:2109 (2000).

3. Kyritsis, D. C., and Gomez, A., "Scalar Dissipation Rate Measurements in Vortex Perturbed Counterflow Diffusion Flames," paper 120, U.S. Sections Second Joint Meeting of the Combustion Institute, Oakland, CA, March 25-28, 2001.

4. Santoro, V. S., Kyritsis, D. C., and Gomez, A., Proc. Combust. Inst. 28:1023 (2000).

5. Pitsch, H., Chen, M., and Peters, N., Proc. Combust. Inst. 27:1057 (1998).

6. Smooke, M. D., Ern, A., Tanoff, M. A., Valdati, B. A., Mohammed, R. K., Marran, D. F., and Long, M. B., Proc. Combust. Inst. 26:2161 (1996).

7. Li, S. C., and Williams, F. A., Combust. Flame 118:399 (1999).

8. Im, H. G., Chen, J. H., and Chen, J., Combust. Flame 118:204 (1999).

9. DeCroix, M. E., and Roberts, W. L., Combust. Sci. Technol. 146:57 (1999).

10. Engleman Jr., R., Rouse, P. E., Peak, H. M., and Baiamonte, V. D., Beta and Gamma Systems of Nitric Oxide, Los Alamos Scientific Laboratory report LA-4364, Los Alamos Laboratories, NM, 1970.

11. Carter, C. D., and Barlow, R. S., Opt. Lett. 19(4):299 (1994).

12. Paul, P. H., Gray, J. A., Durant, J. L., and Thoman, J. W., Appl. Phys. B 57:249 (1993).

13. Drake, M. C., and Ratcliffe, J. W., Chem. Phys. 98:3850 (1993).

14. Smooke, M. D., Crump, J., Seshadri, K., and Giovangigli, V., Proc. Combust. Inst. 23:463 (1990).

15. Bowman, C. T., Hanson, R. K., Davidson, D. F., Gardiner Jr., W. C., Lissianski, V., Smith, G. P., Golden, D. M., Frenklach, M., Wang, H., and Goldenberg, M., GRI-Mech Ver. 2.11, 1995, www.gri.org.

16. Bozelli, J. W., and Dean A. M., Int. J. Chem. Kinet. 27:1097 (1995).

17. Hewson, J. C., and Williams, F. A., Combust. Flame 117:441 (1999).

18. Seshadri, K., and Peters, N., Combust. Flame 73:23 (1988).

19. Takeno, T., and Nishioka, M., Combust. Flame 92:465 (1993).

20. Nishioka, M., Nakagawa, S., Ishikawa, Y., and Takeno, T., Combust. Flame 98:127 (1994).

21. Uludogan, A., Foster, D. E., and Reitz, R. D., SAE technical paper 96-2056.

\section{COMMENTS}

Wolfgang Bessler, Heidelberg University, Germany. How was the NO LIF signal quantified? Especially, which calibration technique was used to derive quantitative mass fractions from the (temperature and quenching corrected) LIF data? Also, what is the estimated experimental error in the resulting NO mass fractions?
Author's Reply. The process of quantification of the NO signal is well established and described in the text with relevant references (Refs. [10-13] in paper). NO laser-induced fluorescence signals are fairly strong. In addition, data are acquired in a phase-locked mode, which can increase the signal-to-noise to any desired level. As a result, for the experimental data reported in the paper, the error bars are smaller than the symbols. 